\title{
D'Alembert: Between Newtonian Science and the Cartesian Inheritance
}

\section{Agamenon R. E. Oliveira}

Polytechnic School of Rio de Janeiro, Federal University of Rio de Janeiro, Rio de Janeiro, Brazil

Email: agamenon.oliveira@globo.com

How to cite this paper: Oliveira, A. R. E. (2017). D'Alembert: Between Newtonian Science and the Cartesian Inheritance. $A d$ vances in Historical Studies, 6, 128-144. https://doi.org/10.4236/ahs.2017.64010

Received: August 25, 2017

Accepted: December 3, 2017

Published: December 6, 2017

Copyright (C) 2017 by author and Scientific Research Publishing Inc. This work is licensed under the Creative Commons Attribution International License (CC BY 4.0).

http://creativecommons.org/licenses/by/4.0/

\begin{abstract}
This paper is a tribute to the tercentenary of d'Alembert's birth. It studies the way how d'Alembert (1717-1783), with his Cartesian education, assimilated and developed Newtonian science. His Cartesianism involved the conception of the intelligibility and rationality of the principles of knowledge. His discovery of Newtonian science determined the direction of his work, with the laws of dynamics and gravitational attraction, as well as the mathematical-physics approach to mechanical problems. However, d'Alembert's work is not just a mere development of Newtonian physics, but a real conceptual reorganization of mechanics, using differential and integral calculus formulated by Newton (1642-1727) and Leibniz (1646-1716).
\end{abstract}

\section{Keywords}

History of Mechanic, History of Mathematics, French Enlightenment, Philosophy of Enlightenment

\section{Introduction}

Jean Le Rond d'Alembert is generally regarded as one of the first great representative figures of Newtonian physics on the European continent during the eighteenth century together with Leonhard Euler (1707-1783) and Alexis Clairaut (1713-1765), both followers of Newtonianism in mathematics and physics. These three mathematicians are the authentic heirs and followers who used mathematical analysis and worked with the mathematization of mechanics (Paty, 2001).

Newtonian mechanics was established in opposition to the Cartesian conception associating mechanics with geometry and the theory of "universal attraction" acting at a distance, contradicting the Cartesian theory of vortices based on subtle matter (Blay, 1995). 
D'Alembert's Newtonianism, especially in relation to his mathematical and physical thought does not deny its Cartesian influences, notably concerning the question of knowledge in general (Blay, 2002: pp. 305-310). It is worth emphasizing that even Newton himself was influenced by Cartesian thought, in spite of his living opposition that appeared in his Principia, whose full title, Principia Mathematica Philosophia Naturalis, opposed the Cartesian Principles of Philosophy.

The opposition between Newton and Descartes (1596-1650) is much clearer in the Principia's demonstrations of the laws of celestial motions, where Newton refutes the theory of Cartesian vortices, and in this sense, the motivation to introduce the second book is to prepare and to build a definitive refutation of that theory. This is also the means to enlarge the acceptance and the defense of a non-natural cause for attraction at a distance. Newton's arguments are indeed sufficiently strong, surpassing the Cartesian objection that accuses Newton of trying to bring "hidden qualities" back to life.

The relations that would be established between Newton's laws and d'Alembert's three principles of movement can be seen as a transition and also a reorganization, obviously implying significant modifications, but never a negation (Paty, 1998). This reorganization of mechanics accomplished by d'Alembert, which appears in his Traité de Dynamique (1743), can be summarized in one statement: Cartesian intelligibility within a Newtonian program (Paty, 2001).

Nevertheless, Leibnizian differential calculus allows d'Alembert to think of mechanical concepts in terms of differential quantities, to arrange them in a homogeneous fashion, and to relate them to the laws of motion (Paty, 2004).

\section{D'Alembert's Biographical Note}

D'Alembert was born on November 17, 1717 in Paris and died on October 29, 1783, also in Paris (see Figure 1). He was abandoned by his parents on the steps of Saint Jean le Rond church, and adopted by an artisan family (Paty, 1993). In spite of this, he received a good education with the support of his biological father, Chevalier Destouches-Canon. With multiple influences on his education such as Jansenism, Malebranchism, and Cartesianism, mathematics drew his attention since the beginning.

He made his acquaintance with the new analysis and the calculus, studying Bernoulli's work and Newtonian science. In 1739, he submitted his first work to the Academy of Science, followed by many others justifying his entrance to the Academy in 1741 as an "Associate adjunct astronomer". Since the 1760's he held a key position in the Academy of Sciences.

He went through a period of almost twenty years of very intense work, culminating with the publication in 1743 of his Traité de dynamique, meaning the general theorem of dynamics or d'Alembert's principle, a kind of unification of mechanics, publishing Traité déquilibre et du movement des fluids the following year, and in 1752 Essay d une nouvelle theorie des resistances des fluides, in 


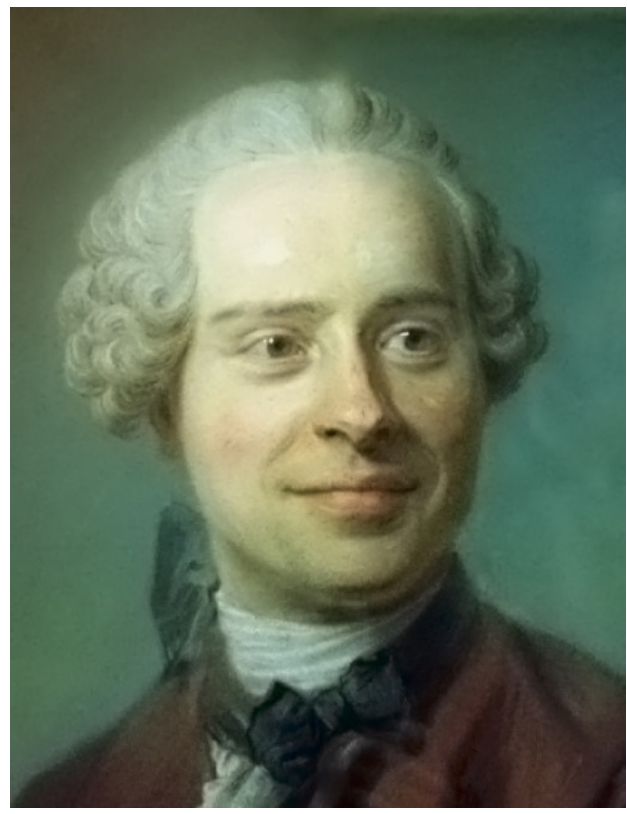

Figure 1. Jean Le Rond d'Alembert (1717-1783).

which hydrodynamics became a branch of mechanics. In 1745, he tackled the three-body problem within Newtonian gravitational theory. In 1749 Recherches sur la precession des equinoxes appeared, followed by Recherches sur points importants du systéme du monde in 1754.

With all these works d'Alembert, Euler, and Clairaut before Lagrange (17361813) and Laplace (1749-1827), became the successors and followers of Newton in the eighteenth century in mechanics and astronomy (Paty, 2002).

In 1747, in mathematics, d'Alembert created and developed a new branch of differential calculus, partial differential calculus, when he postulated a general solution for the wave equation (Paty, 2002: pp. 237-258).

In 1746, in his Reflexions sur la cause general des vents (1746-1747), he presented the first demonstration of the fundamental theorem of algebra, meaning that an algebraic equation of $\mathrm{n}$ degree has $\mathrm{n}$ possible roots real or imaginary.

The Encyclopedia project, also called a Systematic Dictionary of the Sciences, Arts and Crafts, was completed upon its publication in France between 1751 and 1772. It was edited by Denis Diderot (1713-1784) and co-edited by d'Alembert until 1759. It is important to note that the original idea was the translation into French of Ephraim Chamber's Cyclopaedia. The latter had first published his Cyclopaedia, or a Universal Dictionary of Arts and Sciences in two volumes in London in 1728 . This project was replaced by a new work under the general editorship of Denis Diderot. D'Alembert became editor of the mathematical and scientific articles. In fact, he not only helped the general editorship but wrote articles on other subjects and also wrote the Discours préliminaire which introduced the first volume of the Encyclopédie in 1751. This introduction is a general view of eighteenth century knowledge showing its development as well as the multiple connections among the various branches of the scientific knowledge 
(D'Alembert, 2000).

In 1761, he began to publish his Opuscules mathématiques, a work with nine volumes.

He advanced the theory of functions by introducing the concept of arbitrary functions for the solution of differential equations and developed a new method in limit notion trying to clarify the conceptual basis of the mathematical analysis, postulating new theorems of series.

He advanced the theory of functions by introducing the concept of arbitrary functions for the solution of differential equations and developed a new method in limit notion trying to clarify the conceptual basis of the mathematical analysis, postulating new theorems of series.

His mathematical work cannot be separated from his physical studies, especially the application of partial differential calculus to hydrodynamics problems. These studies in particular are an amplification of mechanics by applying the material point method to the mechanics of continuum media, which would be expanded in the following century.

\section{Intellectual Heritage and Education}

D'Alembert's scientific style is essentially characterized in mathematics and physics by the use of mathematical analysis in the modern sense. This means the application of differential and integral calculus from the first quarter of the eighteenth century in the Cartesian sense (Allard, 1963). The importance to him of the Cartesian mathematical heritage can be found in Part II of Discours préliminaire: But above all what immortalized the name of this great man is the application he was able to make of algebra to geometry, one of the greatest and most fortunate ideas that the human mind has ever had. It will always be the key to the most profound investigations, not only in sublime geometry, but also in all the physics-mathematical sciences.

D'Alembert also emphasizes, in the same part of Discours préliminaire, the relevance of the new physics created by Descartes: Let us recognize, therefore, that Descartes, who was forced to create a completely new physics, could not have created it better, that it was necessary, so to speak, to pass by way of vortices in order to arrive at the true system of the world and that if he was mistaken concerning the laws of movement, he was the first, at least, to see that they must exist.

As we know, the first developments of Leibnizian differential calculus and Newton's calculus of fluxions were accomplished in Europe, initially by Leibniz who invented the rules for the calculus, the notations for the differential elements, and the integral symbol. His followers were his disciples from Basel, such as the brothers Jacques (1654-1705) and Jean Bernoulli (1667-1748). Later, came the contributions of Marquis de l'Hôpital (1661-1704), author of Analyse des infiniment petits pour P intelligence des lignes courbes, from 1696, and Pierre Varignon (1654-1722) who developed the differential notations for the velocity and 
acceleration, with the collaboration of Malebranche's group. Also worth mentioning is Fontenelle's work Elements de la geométrie de Pinfini, which appeared in 1727, addressed to clarify the meaning of infinitesimal quantities (Oliveira, 2013: pp. 102-105).

In the Collège des quatre nations D'Alembert was taught elementary notions of mathematics. However, it was by reading books that he learned geometry and infinitesimal analysis. Hence, since the age of nineteen he had familiarity with mathematical analysis. With respect to physics, at that time in his College, it was less advanced, more speculative, while Newton's work was absent. Jean Bernoulli was one of the many influences on d'Alembert. He did not know him personally, but many problems studied by d'Alembert, especially in his Traité, were inspired by Jean Bernoulli.

Malebranche's disciples had the tendency to defend pure analysis, despite immediate applications in kinematics. Newton's physics was ignored for a long time. Later he arrived in France and continental Europe with the laws of motion, the theory of gravitational attraction, and the application of motion laws to planets, or to the "world system". Newton's theory of light and optics also arrived early on the continent. This introduction of Newtonianism on the continent was the result of great effort on the part of Pierre Louis Moreau de Maupertuis (1698-1759) and Alexis Clairaut. Also of importance was Voltaire (1694-1778). In Part II of Discours préliminaire, d'Alembert emphasizes the reception of Newtonianism in France as follows: Maupertuis believed that one could be a good citizen without blindly adopting the physics of one's country, and we ought to be grateful to him for the courage he had to display in attacking that physics. In the same text, he adds: In fact our nation, which is singularly eager for novelties in matters of taste, is on the contrary very much attached to ancient opinions in matters of science.

With evidence from many experiments about the shape of the earth confirming and proving Newtonian ideas against the postulations of Jacques Cassini (1677-1756), director of the Paris Observatory, Newtonianism became established in European continent and it was accepted and adopted by Paris Academy of Sciences where Clairaut and Maupertuis were members. Soon afterwards Newton's "natural philosophy" was received by the public in general. D'Alembert read around 1739 the first volumes of the new Latin edition of the Principia. This edition was published in Geneva in four volumes, in the period 1730-1744, prepared by the priests Thomas Le Seur (1703-1770) and François Jaquiert (1711-1788), with many comments and foot notes emphasizing the geometrical approach used by Newton, as well as his calculus of fluxions.

\section{Genesis of D'Alembert's Principle}

The Traité de dynamique (Firode, 2001), in addition to d'Alembert's works on fluid mechanics and astronomy, are built and developed through differential calculus. D'Alembert's style was thus determined by the use of Newton and Leibniz methods, resulting in a fusion of Newtonian concepts and Leibnizian 
symbolism (D'Alembert, 1921). With respect to the "dynamics theorem", it is important to underline its narrow association with mathematical analysis, and the mechanical conceptions and formulations supported by the tools provided by differential and integral calculus. Therefore, there is a strong link between the differential construction of the quantities that describe the motion and the statements of mechanical principles.

Looking at a genesis of the "d'Alembert's principle" or the "theorem of dynamics", it is possible to identify that it came from three "principles of the motion of bodies" and can be seen as a synthesis of these principles, giving us a "general principle of dynamics", applied to any system of bodies, with constraints or not.

D'Alembert's principle was initially formulated for solid bodies and it is accepted that it was inspired by considerations about composed pendulums. Access to the early hydrodynamics of the works d'Alembert presented to the Academy of Sciences in 1741-1742, before Traité de dynamique published in 1743, has led historians to a new vision of the Traitês genesis (Fraser, 1985: pp. 31-61).

Afterwards, in 1744, d'Alembert published his Traité d' équilibre et de movement des fluids, which dealt with the problem of fluid resistance, based on work done accomplished in 1741-1742, which was used as an "application of his principle of dynamics".

We believe that d'Alembert's famous principle was suggested to by this approach, which can be used as a generalization for any kind of body, and it was conceived by him when studying the problem of a solid body passing throughout a fluid and the resistance of the fluid to this passage. This interaction between the fluid and the body and the consequent resistance implies the annulation of virtual motions (differential motions), corresponding to compensatory actions in opposite senses: this also suggests an approximation with the composed pendulum problem. The motions that are compensated inside the liquid appear equivalent to constraints on solid bodies. The representation of the constraint effect as the annulation of virtual opposite motions is the central idea of his principle of dynamics.

\section{Expressing Physical Quantities Mathematically}

In his Traité de dynamique d'Alembert clearly expressed for the first time his conception of physics. In this work can be found many of his achievements in solid and fluid mechanics (hydrodynamics), and theoretical astronomy, including the first solutions of problems involving three-body gravitational attraction. All of these solutions were of great importance for the future of science due to the results obtained, the methods adopted, the theoretical innovations, and the conceptual propositions, associated with the intelligibility principles involved (Paty, 2004).

The Traite is based on the conviction that what is intelligible in the variable motion of bodies is the movement itself, as the displacement in space over some 
elapsed time, and not what generates this motion, and in addition it is around this idea that dynamics can be organized. The term "dynamics" should be understood as not being directly related to forces but rather to their effects. If force is a cause of motion, like every causes it can escapes to us: in this sense d'Alembert avoids the use of the Newtonian notion of force and prefers to focus on effects. Only the effects, which means variations in motion, are capable of being conceptualized and the laws of these variations are expressed in terms of quantities that describe motion: distance and time, velocity, acceleration, etc., without the use of mass conception through the quantity of motion $(\mathrm{mv})$ and it element $m d v$.

When d'Alembert defines force, it is always in the sense of quantities that express the state of motion (inertia force, for instance) or the effect of the motion variation (motive force or acceleratory force, which appear in Principia).

In the Traité, motive force designates the product of mass by the velocity element, while acceleratory force is the variation of the velocity itself, or its "element" $d v$ divided by the unit of time element, that is $d v / d t$, one quantity purely kinematics. Acceleratory force is actually acceleration.

Time is a fundamental quantity for d'Alembert, defining motion properly, and is the physical variable par excellence for comparing actual motion and the mere displacement of figures, which made mechanics different from geometry. Time variation was the first characteristic of differentiation between mechanics and physics. As stated by d'Alembert, mechanics is geometry within time, it is a kind of geometry with four dimensions, in addition to the three spatial dimensions. Another difference between mechanics and geometry appears because bodies that have some spatial extension are characterized by properties that escape geometry as mass, the impenetrability and gravitational attraction. These properties also escape a priori reasoning, and knowledge of them is obtained through our experience with bodies.

The systematic character and his manner of thinking about mechanical quantities according to their mathematical form (geometrical and algebraic) and more precisely their differential and integral forms, is an important and specific characteristic of d'Alembert's work. It is easy to see this form of thinking in the three initial chapters of his Traité for establishing the fundamental principles of his science of movement and consequently defining concepts and quantities and how to relate them.

\section{A New Look at Newton's Second Law}

In D'Alembert's dynamics (D'Alembert, 1921), the fundamental law is not Newton's second law, which establishes the proportionality between variations of motion and impressed motive force. Rather Newton's three laws are replaced by "principles of motion", which are originary or founding principles, from which is excluded any external notion of cause as force, for instance. Newton's second law, which can be written as $F=\Delta(m V)=m \Delta V$, only received its diffe- 
rential form $F=m d V / d t$ in 1750, an accomplishment of Euler.

D'Alembert also included a formula for acceleration, representing the variation of motion, at the beginning of his famous Traité, when describing uniform motion he describes accelerated motion in a different sense. Step by step he builds up the relationship that permits the change from uniform motion to accelerated motion, by means of a graphical representation in two dimensions, space and time, in which the vertical axis represents a uniform motion of reference that corresponds to time $t$; $\mathrm{e}$ is the horizontal axis representing the spaces that correspond to the position variable. Uniform motion corresponds to an inclined straight line with respect to the first axis (see Figure 2); non-uniform motion is characterized by a nonlinear relationship between space and time, represented by a curve $e=e(t)$, convex or concave, according to the type of motion, accelerated or decelerated. By analyzing this curve, d'Alembert defines the quantities that characterize non-uniform motion, velocity, acceleration and other derived quantities. In this study it is not necessary to know that "this continuum variation only can result because an external cause acts continuously".

The definition of instantaneous velocity at a time $t$ in the way proposed by d'Alembert introduces, at the same time, the differential element of the space variable $d e$, conceived as the unit of time, with being equal to $d t$ and here assumed to be constant with time; the notion of virtual velocity, not even mentioned in this part of the book. The meaning of instantaneous velocity is one uniform velocity during the element of $d t$ duration, with value and direction immediately before this instant; it is taken from the curve tangent from the diagram $e=e(t)$ : the velocity is defined by $u=d e / d t$.

Similarly, d'Alembert builds acceleration by abstracting velocity and taking into account (virtual) motion, i.e., the effect whose cause is motion variation and the correspondent spaces. D'Alembert's reasoning led him to evaluate greater or lesser displacements with respect to uniform motion, by means of the distance in the space axis, described by the moving point in time $t$, between the curve and the tangent.

Working with definitions, made with the use of a diagram containing spaces and times corresponding to a specialization of time and the geometrization of these two quantities, d'Alembert presents the first consequences of his program

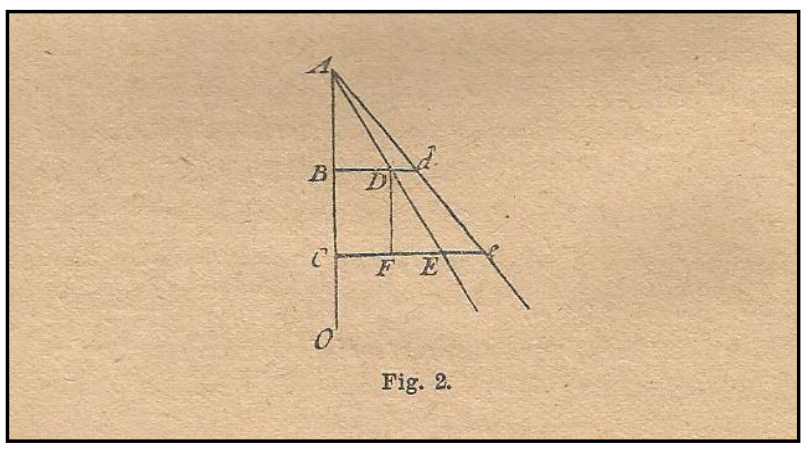

Figure 2. Representation of uniform motion. 
regarding a new dynamics that considers "motion is only motion".

The "inertia principle", occupying the first place, establishes that motion is naturally uniform, which allows time to be geometrized, and variable motions to be defined by taking into account deviations from the uniformity: building "the geometrical quantities of motion", for uniform and variable motion, using the "differential element" of these quantities.

From these theoretical developments, D'Alembert obtained the means to think of motion and its variations in terms of the originary principle of movement, in addition to the inertia principle, the motion composition principle, and the equilibrium principle. The law of motion composition (velocities) has a corollary for use with acceleration: in the change of motion that occurs in one such instant $t$, the velocities previously acquired (due to the inertia principle) can be composed (geometrically) with the "elements" (the differentials) of the velocities impressed. It is possible to write one velocity directly after its variation (increase or decrease), as $V+d V, d V$ being positive or negative and taking the intensity and direction.

Similarly, the equilibrium principle is conceived as the annulation of the total velocity of the many elements that the system contains (or more exactly the quantity of motion) and thus can provide the notion of virtual velocity, which can be understood as a kind of tendency contrary to motion and whose differential element of velocity is an "intuitive" representation that seems to be behind d'Alembert's considerations.

\section{D’Alembert's Principle}

The principle is not a new fundamental principle, as the three fundamental principles postulated by d'Alembert are: the principle of inertia force, the principle of motion composition, and the principle of equilibrium. It is a kind of derived principle obtained by using the second and third principles. Later d'Alembert called it a second order principle, while sometimes it was understood as a simple method.

Its starting point is based on the observation that in any mechanical interaction among bodies, the initial motions acquired by each body, independent of their relations with others, can be decomposed by means of the second principle into other two. First motion is what is actually acquired by means of mutual interactions and second motion what is received or lost through the action of other bodies.

Thus, in problems of dynamics, in any phenomenon of motion transmission we can introduce a general method in order to solve dynamical problems by means of d'Alembert's theorem (Fraser, 1985: pp. 145-159). To obtain resulting motions due to mutual action among bodies it is sufficient to decompose impressed motions received initially by them in two systems of motions: one encompassing actual motions (to be determined) which are compatible, i.e., they do not feed themselves reciprocally; the second by the motions received or lost 
due to the action of other bodies.

Then the principle is based on the fundamental idea that the equilibrium conditions of the received or lost motions necessarily determine the laws to be obeyed by the transmission of motion, i.e., by the dynamical process itself.

D'Alembert's principle appears in Part two of the Traité (see Figure 3) with the following statement:

\subsection{General Principle}

Given a system of bodies arranged mutually in any manner whatever, let us suppose that a particular motion is impressed on each of the bodies that it cannot follow because of the action of others, to find that motion that each body should take.

\subsection{Solution}

Let $A, B, C$, etc. be the bodies composing the system, and let us suppose that the motions $a, b, c$, etc. be impressed on them, and which be forced because of the mutual action of the bodies to be changed into the motions $a^{\prime}, b^{\prime}, c^{\prime}$, etc. It is clear that the motion a impressed on the body $A$ can be regarded as composed of the motion a' that it takes, and of another motion $\alpha$; similarly, the motions $b, c$, etc. can be regarded as composed of the motions $b, \beta ; c, \chi$; etc.; from which it follows that the motions of the bodies $A, B, C$, etc. would have been the same, if instead of giving the impulses $a, b, c$, one had given simultaneously the double impulses $a^{2}, \alpha ; b, \beta ; \mathcal{C}^{2}, \chi ;$ etc. Now by supposition of the bodies $A, B, C$, etc. took among themselves the motions $a^{\prime}, b$, $c$, etc., that is, that if the bodies had received only the motions $\alpha, \beta, \chi$, etc., these motions would have destroyed each other and the system would remain at rest.

From this results the following principle for finding the motion of several bodies which act on one another. Decomposing the motions $a, b, c$, etc., impressed

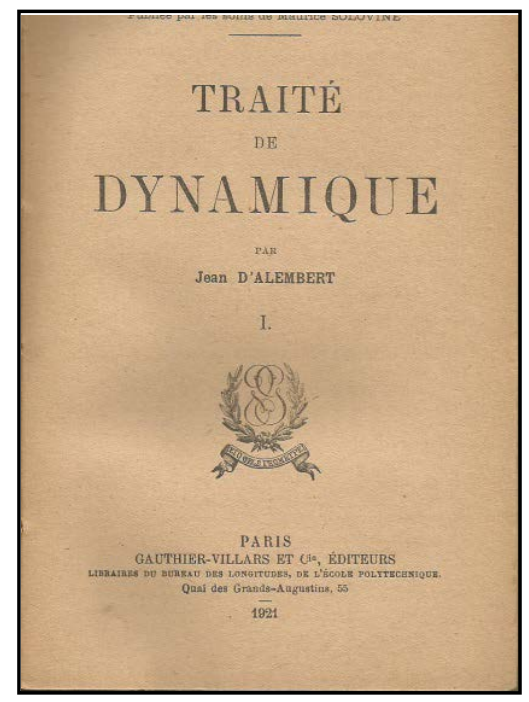

Figure 3. Traité de Dynamique. 
on each body into two others $a^{\prime}, \alpha ; b, \beta ; c^{\prime}, \chi$; etc., which are such that if the motions $a^{\prime}, b, c$, etc., were impressed alone on the bodies they would retain these motions without interfering with each other, and that if the motions $\alpha, \beta, \chi$ were impressed alone, the system would remain at rest, it is clear that $a^{\prime}, b^{\prime}, c^{\prime}$ will be the motions that the bodies will take by virtue of their action.

D'Alembert's principle can be correctly understood if we also look at the principle of virtual velocities, which consists of considering a given system with several bodies that can be reduced to points being acted upon by any kind of force. If we apply a small motion to the system, each body is displaced an infinitesimal space. If we multiply each force by the displacement of its points of application and add them for the whole system, the result is zero.

For the dynamical case, we can consider that the velocities of each body can be decomposed in three fixed and perpendicular directions; the decrease of these velocities will represent the motions lost along the same directions and their increase will be the motions lost in the opposite directions. Therefore, these lost motions will be expressed, in general, by the mass multiplied by the element of velocity and divided by the time element and they will have contrary directions to the velocities. Using this approach, it is possible to obtain a general formula to represent the motions of bodies which will provide a solution for any dynamical case. This is clearly demonstrated in the next item.

\section{Application of D'Alembert's Principle}

One of the fundamental objectives postulated by d'Alembert in his Traité de Dynamique is the provision of a general method to solve mechanical problems without any kind of metaphysical resource. At that time, it was believed that the principle of vis viva conservation could not do this. In addition, the methods derived by d'Alembert's principle also replaced all types of particular approach with a general methodology.

In this paper we shall present d'Alembert's solution which appears in the second part of his Traité, analyzing the simplest problem, Problem I, a composed pendulum (see Figure 4).

\subsection{Problem I}

Find. the velocity of a bar $C R$ pivoted in $C$ (Figure 22 in Traite) containing the bodies $A, B, R$, supposing that these bodies describe in equal time the infinitesimal arcs $A O, B Q, R T$, perpendicular to the bar.

In fact, the problem proposed by d'Alembert is a massless rigid bar with concentrated masses in $A, B$ and $R$. He remarks that the main difficulty of the problem is to discover the line $R S$, described by one body, for instance $R$ in the same time in which it has displaced $R T$; then the velocities $B G, A M$ and in any other point or bodies attached will be known. If we look at the impressed velocities $R T, B Q, A O$ it is easy to see that they are composed by the velocities $R S, S T ; B G$, $-G Q$; $A M,-M O$; using the lever principle, $C A R$ will stay in equilibrium, if the 


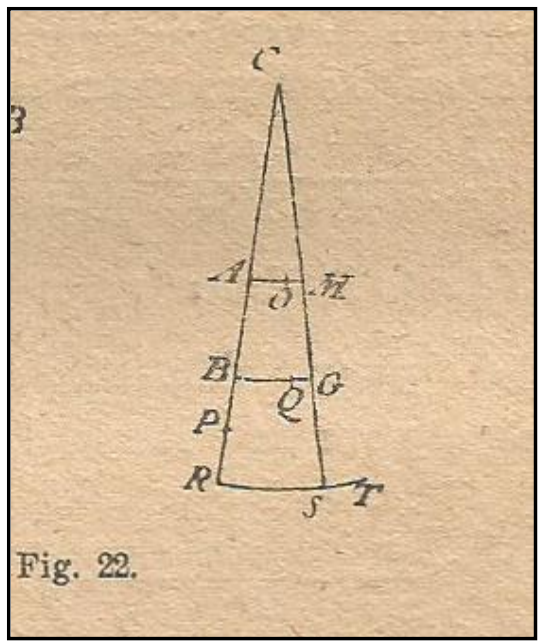

Figure 4. Problem I in Traité (Figure 22).

bodies $R, B, A$, receive only the motions $S T,-G Q,-M O$. Then, one writes:

$$
A \cdot M O \cdot A C+B \cdot G Q \cdot B C=R \cdot S T \cdot C R
$$

Adopting: $A O=a ; B Q=b ; R T=c, C A=r, C B=b ; C R=\rho ; R S=z$.

One can write:

$$
R \cdot(c-z) \cdot \rho=\operatorname{Ar}(z r / \rho-a)+B r(z r / \rho-b)
$$

Which yields:

$$
Z=(A a r \rho+B b r \rho+R c \rho \rho) /\left(A r^{2}+B r^{2}+R \rho^{2}\right)
$$

\subsection{Corollary}

If the forces impressed in $A, B, R$, are respectively $F, f, \varphi$, we can find the accelerating force of the bar, as follows:

$$
(F r+f r+\varphi \rho) \cdot \rho /\left(A r^{2}+B r^{2}+R \rho^{2}\right)
$$

Using $a, b, c$ the values $F / A, f / B, \varphi / R$, it is possible to introduce the differential arc $d s$ element described by the radius $C R$, and $u$ as the velocity of $R$, to arrive at the general formulation:

$$
(F r+f r+\varphi \rho) \cdot \rho \mathrm{d} s /\left(A r^{2}+B r^{2}+R \rho^{2}\right)=u \mathrm{~d} u
$$

For any kind of forces $F, f, \varphi$.

The velocity $u$ is given by:

$$
u^{2}=2 \int(F r+f r+\varphi \rho) \cdot \rho \mathrm{d} s /\left(A r^{2}+B r^{2}+R \rho^{2}\right)
$$

Obviously, the complexity of solving (6) depends only how the forces $F, f, \varphi$ can vary. If the forces are all constant over time and position the solution is easily obtained. In the case that $F, f, \varphi$ can vary with time or position and the expression (6) can become difficult to solve. 


\section{The D'Alembertian Heritage for Lazare Carnot's Mechanics}

In Principes fondamentaux de Péquilibre et du mouvement, Carnot (1753-1823) explains in a more general way his ideas about mechanics (Carnot, 1803): There are two ways to see mechanics and its principles. The first one is by considering it as a theory of forces, the causes that impress motions. The second is by considering it as a theory of motions by themselves. In the first case, we can establish the rationality about the causes in any situation, which impress or tend to impress motions on bodies. In the second case, we see the motion already impressed, acquired or belonging to bodies; and thus we search only for what are the laws according to which these motions appear and propagate, being modified or being destroyed in each circumstance. Each one of these two ways to study mechanics has its advantages and disadvantages. The first way is generally considered the simplest, but it has the disadvantage of being founded on the obscure metaphysical notion of what a force is. Therefore, what clear idea can present to spirit in this subject the name of cause? There are so many types of cause! What can we understand in the precise language of mathematicians by one force, i.e. by a double cause or a triple of one other?... These causes are the willing or the physical constitution of a man or of an animal that by means of its action produces the motion? But what is a double willing or a triple one of another willing?

If we adopt the position of not distinguishing the cause from the effect, in other words, if we understand by the word force the quantity of motion even if it arises in the body in which it is applied, become intelligible, but then we go back to the second way to study the question, that is, mechanics really is a theory of communication of motions.

As the above quotation expresses, Carnot's mechanics were directly influenced by d'Alembert. Actually, Carnot adopts the second way mentioned above in order to overcome the difficulty in identifying the force. By doing this Carnot adopts an analytical tradition which comes from Descartes and especially from d'Alembert, also influenced by Lagrange and others. As a consequence of the second way adopted by Carnot, mechanical laws become the laws of communication of motion, which is also his understanding of how a machine operates. His contribution to applied mechanics is a natural consequence of his machine visualization or a system for communicates motion. Carnot's theory of machines is the study of these forms for the communication of motion (Oliveira, 2013).

The similarities between the mechanics of d'Alembert and Lazare Carnot are not restricted to their conceptual foundations, as we have seen above for the case of the concept of force. They also use a similar structure in terms of the principles on which mechanics is anchored. In his Traité de dynamique, d'Alembert chooses three principles as the foundations of dynamics: the principle of inertia force, the principle of motion composition, and the principle of equilibrium. The correct understanding of this second principle is of fundamental importance to 
have a deeper vision of Carnot's mechanics. For this, it is also necessary to return to d'Alembert principle.

If we examine Carnot's Essais, it can be seen that he uses only two principles to present his mechanics: the principle of equality between action and reaction and the principle of nullity of the relative motion subsequent to a shock (between hard bodies). Obviously the first principle is different from Newton's because of their different conceptions of force. This principle to Carnot is much more than a law of equality between two quantities of motion. In the second principle presented, he uses motion decomposition as did d'Alembert in his famous principle. The two motions considered are impressed motion and attained motion, because the difference between them is exactly the force that a body would have if it had not received the impressed motion.

To understand the decomposition of velocities used by Carnot, the following diagram is shown (see Figure 5).

Carnot's original contribution is his association of geometric motion with d'Alembert's decomposition of velocity. He defines geometrical motion as anything compatible with a body's constraints. In the above diagram, if we consider a body colliding with a plane, if $W$ is the body velocity before colliding with the plane which coincides with direction of $V$, only it is reversible, because it maintains its system constraints. $U$ is not permitted, because the body would penetrate the plane, which is by definition impossible, consequently this motion is not reversible (geometric).

The first references to Carnot's work appeared in the first decades of the nineteenth century. This was in the work of André Guenyveau (1782-1861), who in 1810 published the Essai sur la science des machines, different from Carnot's works. But Guenyveau does not attributes to Carnot the origin of the principle, even though in the preface of his Essai, Carnot is referred as an author of a general treatise on machines.

Jean-Nicolas-Pierre Hachette (1769-1834) is another author who cites Carnot's Principes fondamentaux de Péquilibre et du mouvement, appearing in the preface of his Traité élémentaire des machines, published in 1811. Hachette

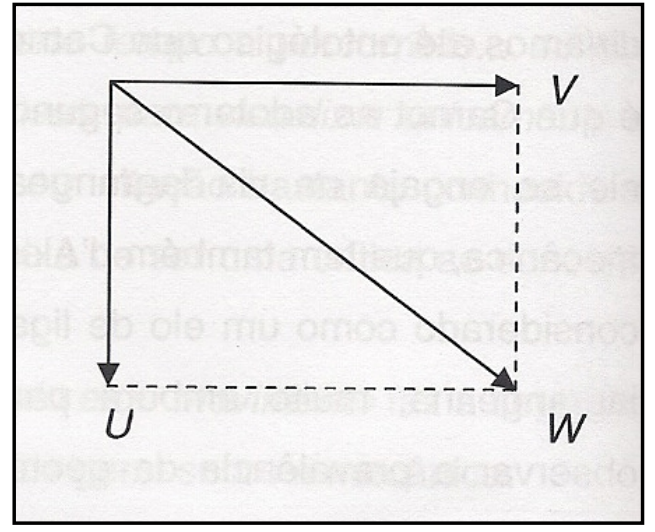

Figure 5. The principle of motion decomposition. 
remarks that Carnot studied in the last chapter of his Principes fondamentaux, the whole theory of machines and moving applied forces. In addition, he calls Carnot the most profound savant and experienced engineer. Paradoxically Hachette uses few of Carnot's achievements. In fact, the course he taught is much more a collection of drawings of particular machines, as well as studying machine elements, such as gears, pulleys, etc.

Navier (1785-1836) made an evaluation of Carnot's contribution to machines, discussing the Essai sur machines, published in 1783 and the Principes fondamentaux de Péquilibre et du movement, which appear in 1803. Navier considers that Carnot creates a general theory of machines, thoroughly based in mechanical principles.

Finally, it should be noted that d'Alembert and Carnot see actions among bodies by means of shocks instead of continuous interactions because these actions are realizable and become evident through their effects. However, it is worth emphasizing that Carnot broke with the essentially rationalist tradition that came from d'Alembert and ran through Lagrange, in which mechanics is seen as a branch of pure mathematics and experience was a kind of legitimation that in principle was purely rational. A kind of empiricism which came from English empiricism, while Newton would be adopted by Carnot since he continued to attribute to reason a fundamental role within the system of knowledge in establishing the causal nexus in the form of laws.

\section{Final Remarks and Conclusion}

D'Alembert conceives dynamics as a science of changes in bodies' motions and he postulates to express these changes only by means of internal variables of motion. Using this method, he established the possibility of conceiving motion physically, with respect to real causes, without the use of external concepts, such as the concept of force. In spite of this, he states that there is a mutual relationship between physical cause and its effects. This can be expressed by the notion of instantaneous acceleration, associating physical meaning with differential forms of the time quantity and the concept of virtual motion. This construction between a temporal causality and differential analysis, in fact, led him to correlate variables of motion in a form of principles and thus to conceptually reorganizing Newtonian mechanics. Since, for him, the fundamental law of mechanics was not Newton's second law, but the "principle of dynamics", formulated to any system of mechanically interacting bodies. This step was a real impulse towards the total mathematization of mechanics, with the physical character of this science becoming more explicit.

If Newton's three laws are replaced by three "principles of motion", originary and foundational principles, in which all external causes, or forces are excluded. The first is the principle of inertia, establishing motion as naturally uniform, which permitted a geometrization of time as well as the definition of the variation of motion considering differences with respect to uniformity: building up 
the "geometrical quantities" of motion, whether uniform or not, using the "differential thinking" of these quantities. D'Alembert then adds the principle of motion composition and the principle of equilibrium. Using the composition of motion, he takes the acceleration that occurs in a time $t$, the velocities acquired previously (due to the inertia principle), performs the composition (geometrically) with the differential elements of velocities. It is possible to directly write one velocity after its variation $(v+d v)$, taking into account intensity and direction. Similarly, the equilibrium principle is conceived as an annulation of the total velocity of all components of the system (more exactly the quantities of motion). This step allows virtual velocity to be considered, which should be understood as a tendency, opposed to the motion itself.

Indeed, it is the analytical thinking, represented by differential quantities that allow d'Alembert to take Newton's "laws of motion", significantly modify their formulation, and reorganize on this foundation his new manner of thinking about mechanics. This is the meaning of the structure of the Traité de dynamique, which led him to reduce mechanics to the three above mentioned principles: inertia, composition of motion, and equilibrium. With all these transformations inside Newton's theoretical framework, d'Alembert's Newtonianism can be considered as new and creative.

If we look at d'Alembert's scientific trajectory, it is possible to visualize two different but inseparable aspects. One representing a search for the rationalization (Alquié, 1963) of physical phenomena and its laws which frequently followed a theoretical unification based on fundamental principles of a physical nature. A second aspect is a critique, highlighting the notions and conceptions that appear in his analyses. Finally, is the privileged position that mathematics occupies. To d'Alembert among all scientific knowledge, mathematics is the nearest of the pure reason because of the nature of his object itself. In addition, mathematics shows the ways to achieve certainty with respect to reasoning.

\section{Acknowledgements}

I am in debt with professor Michel Paty who sent to me many papers, documents and his D'Alembert's biography. Brazilian historians of sciences consider Paty one of the most important experts on D'Alembert's thought.

\section{References}

Allard, J.-L. (1963). Le mathématicisme de Descartes. Ottawa.

Alquié, F. (1963). Oeuvres philosophiques de Descartes. Paris: Garnier Frères.

Blay, M. (1995). Les “Principia” de Newton. Paris: Presses Universitaires de France.

Blay, M. (2002). La Science du Mouvement: De Galilée à Lagrange.

Carnot, L. (1803). Principes fondamentaux de l équilibre e du movement. Paris.

D’Alembert, J. (1921). Traité de Dynamique. Gauthier-Villars et Cie. Éditions. https://doi.org/10.1259/jrs.1921.0058

D’Alembert, J. (2000). Discours préliminaire de I Encyclopédie (1751). Edité par M. Mal- 
herbe, Paris. Vrin.

Firode, A. (2001). La Dynamiue de d Alembert. Belarmin/Vrin.

Fraser, C. (1985). D'Alembert's Principle: The Original Formulation and Application in Jean d'Alembert's Traité de Dynamique (1743). Part I. Centaurus, 28, 31-61. https://doi.org/10.1111/j.1600-0498.1985.tb00801.x

Fraser, C. (1985). D’Alembert's Principle: The Original Formulation and Application in Jean d'Alembert's Traité de Dynamique (1743). Part II. Centaurus, 28, 145-159. https://doi.org/10.1111/j.1600-0498.1985.tb00834.x

Oliveira, A. R. E. (2013). A History of the Work Concept. From Physics to Economics. Springer.

Paty, M. (1993). D'Alembert, Jean Le Rond, 1717-1783. Dictionaire des philosophes, deuxième éd. Rev. et augm. Paris: Presses Universitaires de France.

Paty, M. (1998). Societé d'Édition Les Belles Lettres.

Paty, M. (2001). D'Alembert, la science newtoniènne et P héritage cartesièn. Corpus (Revue de philosophie, Corpus des oeuvres de philosophie en langue française (No. 3, pp. 19-64.).

Paty, M. (2002). Analyse et Dynamique: Études sur loeuvre de d Alembert. Les Presses de l'Universitté de Laval.

Paty, M. (2002). Les recherches actuelles sur d Alembert. Quebec: Presses de l'Université Laval.

Paty, M. (2004). L'élement differentiel de temps et la causalité physique dans la dynamique de d Alembert. Éditions Peetters. Louvain (Be). 